\title{
Vom gymnasialen Schwerpunktfach zum Studienfach: Bringt ein fachverwandtes Studium mehr Studienerfolg als ein fachfremdes?
}

\section{Maren Oepke}

Das wissenschaftspropädeutische Lernen in den gymnasialen Schwerpunktfächern soll zu einer vertieften Vorbereitung auf verwandte Studienrichtungen in den Hochschulen führen. Inwiefern eine Verwandtschaft von Schwerpunkt- und Studienfach auch Effekte auf Studienerfolg hat, wird im Beitrag anhand einer Stichprobe von ca. 1100 Deutschschweizer Studierenden untersucht. Es zeigt sich, dass Personen, die Fächer studieren, welche inhaltlich ihren Schwerpunktfächern ähneln, im Durchschnitt keine besseren Studienleistungen erbringen als Studierende, die ein dem Schwerpunktfach fremdes Studium absolvieren. Die Ergebnisse werden vor dem Hintergrund des gymnasialen Ziels der allgemeinen Studierfähigkeit diskutiert.

\section{Einleitung}

Die Schnittstelle zwischen Gymnasium und Hochschulen wird in der Schweiz in der öffentlichen Diskussion häufig dahingehend thematisiert, ob die Gymnasien ihre Absolventinnen und Absolventen genügend für den Besuch der Hochschulen zu qualifizieren vermögen. Das Ziel der Studierfähigkeit stellt neben der breiten Allgemeinbildung das zentrale Ziel des Schweizer Gymnasiums dar (vgl. Maturitätsreglement, MAR 95). Gesichert werden soll es über den verpflichtenden Besuch von zehn bis elf Grundlagenfächern, der insgesamt ca. 75 bis 85\% der Unterrichtszeit in Anspruch nimmt. Mit dem Ziel ist die Allgemeingültigkeit des Maturaausweises als Zulassung zu einem frei wählbaren Hochschulstudium verbunden (Ramseier et al., 2005). Eingeführt wurde der obligatorische Besuch der Grundlagenfächer mit der letzten Maturitätsreform von 1995. Die Gestaltung einer damit zumindest formal einheitlichen Matura bedeutete eine Abkehr von der Fakultätsreife, die bis dahin mit den Maturitätstypen für das Schweizer Gymnasium noch prägend war (Brüggenbrock, Eberle \& Oelkers, 2016). Zugleich wurde über die Einführung von Schwerpunkt- und Ergänzungsfächern sowie der Maturaarbeit eine interessengeleitete Individualisierung der gymnasialen Ausbildung ermöglicht. 
Schwerpunktfächer setzen - vergleichbar Leistungskursen in Deutschland den Hauptakzent im persönlichen Bildungsprofil. Mit ihnen ist nicht nur eine wissenschaftspropädeutische und exemplarische Vertiefung in ein bestimmtes Wissensgebiet verbunden, sondern offensichtlich auch eine vertiefte Vorbereitung auf die mit den gewählten Fächern verwandten Studienrichtungen (Eglin-Chappuis, 2007). So fühlte sich in der Befragung der ersten Evaluationsstudie zum MAR 95, EVAMAR I (Ramseier et al., 2005), die Gruppe der Maturandinnen und Maturanden, die ein ihrem Schwerpunktfach inhaltlich ähnliches Studienfach anvisierten, deutlich besser auf ihr Studium vorbereitet als die Gruppe, die ein fachfremdes Studium wählen wollte. Ähnlich schätzten Absolventinnen und Absolventen von für das jeweilige Studienfach atypischen Schwerpunktfächern im dritten Studiensemester ihre eigenen Kenntnisse deutlich tiefer ein, als es der Wichtigkeit für das Studium ihrer Wahrnehmung nach entsprach (Notter \& Arnold, 2006). Inwiefern diese subjektiv wahrgenommenen Vorteile der Gruppe mit schwerpunktfachverwandten Studienrichtungen auch mit objektiv besseren Studienleistungen einhergehen, wurde allerdings bisher für die Schweiz nicht systematisch untersucht. Dieser Frage wird in vorliegendem Beitrag nachgegangen.

Dazu werden auf der Grundlage der Verbindung der Schwerpunktfächer mit den Studienrichtungen zwei Gruppen von Studierenden mit verwandten vs. nicht verwandten Studienrichtungen gebildet. Danach erfolgt ein Gruppenvergleich im Hinblick darauf, ob sich Unterschiede beim Studienerfolg zeigen. Im Folgenden werden zunächst Befunde zur Beziehung zwischen Schwerpunktfach und Studienfachwahl und zur Prädiktion von Studienerfolg referiert, um dann die Forschungsfrage sowie deren Bearbeitung darzustellen und die Ergebnisse zu diskutieren.

\section{Vom gymnasialen Schwerpunktfach zum Studienfach}

Das Schwerpunktfach umfasst ca. 10 bis 15\% der Unterrichtszeit, die Stundendotationen belaufen sich kantonal unterschiedlich auf zwischen 12 und 18 Jahreswochenstunden (JWS), mit einem Mittel von 14.5 JWS (Eberle \& Brüggenbrock, 2013, Stand 2011). Prinzipiell kann aus insgesamt 13 Fächern ausgewählt werden: (a) alte Sprachen (Latein und/oder Griechisch), (b) moderne Sprachen (eine dritte Landessprache, Englisch, Spanisch oder Russisch), (c) Physik \& Anwendungen der Mathematik (PAM), (d) Biologie \& Chemie (B\&C), (e) Wirtschaft \& Recht, (f) Philosophie/Pädagogik/Psychologie (PPP), (g) bildnerisches Gestalten und (h) Musik. Das tatsächliche Angebot ist vom Kanton bzw. der Schule abhängig1.

Gemäss der EVAMAR I-Befragungen von Maturandinnen und Maturanden werden die Schwerpunktfächer vor allem entsprechend den Interessen und den 
schulischen Fähigkeiten gewählt, weniger aufgrund der Ausbildungsmöglichkeiten oder im Hinblick auf das Wunschstudium oder daraufhin, die Noten bzw. den Aufwand für das Fach zu optimieren (Ramseier et al., 2005; ähnlich für die deutschen Leistungskurse: Abel, 2002). Dabei erfolgt die Wahl des Schwerpunktfachs geschlechtsspezifisch. Während Schüler eher «Wirtschaft \& Recht» oder «Physik \& Anwendungen der Mathematik» wählen, belegen Schülerinnen häufiger «Philosophie, Pädagogik \& Psychologie», die musischen sowie die sprachlichen Fächer (Bundesamt für Statistik [BFS], 2016, S. 8). Erklärt werden diese Geschlechtsunterschiede, die sich häufig in geschlechtstypischen Studienfachwahlen fortsetzen, im Rahmen von Berufswahl- und Laufbahntheorien mit geschlechtstypischen Interessen und ihrem Zusammenspiel mit Fähigkeiten und Wertvorstellungen (Gottfredson, 1981), dem Vorhandensein von Rollenmodellen, unterschiedlicher sozialer Unterstützung und geschlechtsspezifischen Selbstwirksamkeits- und Ergebniserwartungen (Eccles, 1994; Lent, Brown \& Hackett, 1994) im Laufe der Sozialisation. Da verschiedenen Befunden zufolge auch für die Studienwahl gilt, dass vor allem nach Interesse und Neigung entschieden wird (für die Stichprobe der vorliegenden Untersuchung: Oepke \& Eberle, 2014; weiterhin z. B. Aeschlimann, Herzog \& Makarowa, 2015; BFS, 2009; Nagy, 2005; Schnabel \& Gruehn, 2000), kommt es etwa in Fortsetzung des Interesses des Öfteren zu inhaltlich verwandten Verbindungen der schulischen mit der universitären Ausbildung. Aufgrund dieser «Koppelung» wird die Schwerpunktfachwahl zum Teil als richtungsweisend für die spätere Studienfachwahl angesehen (z. B. Nagy, Trautwein, Baumert, Köller \& Garrett, 2006; Schweizerische Koordinationsstelle für Bildungsforschung [SKBF], 2014). Vor allem für MINT-Studienfächer liegen Befunde vor, denen zufolge eine Entscheidung für diese Studienrichtungen bereits durch die Wahl des Schwerpunktfachs vorgespurt und stark beeinflusst wird (Berweger, Bieri Buschor \& Keck Frei, 2015; Schnabel \& Gruehn, 2000; SKBF, 2014).

Bezüglich der Enge der Verbindung über alle Studienrichtungen hinweg gesehen existieren unterschiedliche Interpretationen. Die Spanne reicht von einer als «locker» angesehenen Koppelung zwischen dem gymnasialen Bildungsprofil und der Studienwahl (Eglin-Chappuis, 2007; Ramseier et al., 2005) bis hin zur Interpretation als einem «in der Regel starken Zusammenhang» (SKBF, 2014, S. 152). Auch die Natur des Zusammenhangs scheint nicht eindeutig. Gemäss Zwick und Renn (2000) greift dasselbe Fachinteresse, das bereits in die schulische Kursauswahl einfliesst, auch bei der Studienorientierung. Volodina, Nagy und Retelsdorf (2015) zeigen die Bedeutung beruflicher Interessensprofile bei der Vorhersage des Übergangverhaltens auf. Danach etablieren sich diese Interessen schon etwa mit 14 Jahren und scheinen relativ «robust gegenüber schulischen Sozialisationseffekten»(S. 99) zu sein. Möglicherweise wird ein entsprechendes Interesse durch die Schwerpunktsetzung im Gymnasium aber auch erst massgeblich geprägt (Eglin-Chappuis, 2007) oder verstärkt (SKBF, 2014). 
Schliesslich führt der oben bereits erwähnte Aspekt, wonach sich Maturandinnen und Maturanden besonders gut auf inhaltlich verwandte Studienrichtungen vorbereitet fühlen, vermutlich dazu, dass sie in der Absicht einer entsprechenden Studienfachwahl bestärkt werden (Ramseier et al., 2005). Andererseits könnten die Maturandinnen und Maturanden den Eindruck haben, in einem nicht verwandten Fach über nicht genügende Kompetenzen zu verfügen (EglinChappuis, 2007).

Empirisch zeigte sich für die Schweiz, dass Maturandinnen und Maturanden mit Kenntnissen aus Schwerpunktfächern am Ende des Gymnasiums entsprechend bessere Leistungen in Schulleistungstests erbrachten als solche, die nur das Grundlagenfach besucht hatten (Eberle et al., 2008; Schumann, Eberle \& Oepke, 2013). Insofern kann durchaus von einer besseren Vorbereitung auf inhaltlich verwandte Studienrichtungen ausgegangen werden, die zudem mit höherem Studienerfolg einhergehen könnte.

\section{Bedeutung schulischen Wissens für Studienerfolg}

Befunde zeigen, dass schulisches Wissen zur Prädiktion von Studienerfolg häufig gemessen an Studiennoten - beiträgt. Demnach weisen Kenntnistests, die zum Teil Schulwissen, aber auch studienspezifisches Wissen zum Testgegenstand haben (Deidesheimer Kreis, 1997), eine zufriedenstellende prognostische Validität für Studienleistungen auf (z. B. für die GRE Subject Tests: Kuncel \& Hezlett, 2007; für die SAT II Subject Tests: Ramist, Lewis \& McCamleyJenkins, 2001; für den deutschen Sprachraum siehe Hell, Trapmann \& Schuler, 2007). Als noch höher erweist sich vielen Studien zufolge die Vorhersagekraft von Schulnoten, einem Indikator für das Vorhandensein von Wissen, für Studiennoten (z. B. Baron-Boldt, Schuler \& Funke, 1988; Burton \& Ramist, 2001; Linn \& Hastings, 1984; Robbins et al., 2004; Trapmann, Hell, Hirn \& Schuler, 2007), insbesondere jene der Maturadurchschnittsnote, die sich häufig als bester Einzelprädiktor zeigt (Trapmann, 2008). Die Vorteilhaftigkeit von Vorwissen in Bezug auf Studienerfolg scheint für naturwissenschaftliche Studienfächer besonders relevant zu sein: Studien zeigen positive Effekte auf Studienleistungen vor allem zu Beginn naturwissenschaftlicher Studiengänge (z. B. Alters, 1995; Sadler \& Tai, 2001). Dabei wirkt sich offensichtlich nicht nur positiv aus, wenn überhaupt zuvor ein schulischer fachaffiner Kurs besucht wird, sondern verschiedentlich erwies sich eine vertiefte fachliche Auseinandersetzung als förderlicher als Unterricht, der in die Breite ging (Freyer, 2013; Schwartz, Sadler, Sonnert \& Tai, 2008; Tai, Sadler \& Loehr, 2005). Zieht man schliesslich Studienabbruch als einen weiteren Indikator für Studienerfolg in Betracht, so unterstreichen Befunde, wonach sich mangelnde mathematische und naturwissenschaftliche Vorkenntnisse in den Fächergruppen Mathematik/Naturwissenschaften sowie 
Ingenieurwissenschaften als abbruchfördernd erweisen (Heublein, Hutzsch, Schreiber, Sommer \& Besuch, 2009; Oepke \& Eberle, 2016; Rode Larsen, Bjørnøy Sommersel \& Søgaard Larsen, 2013), ebenfalls die Bedeutsamkeit von Vorwissen.

Andererseits schnitten gemäss einer Studie der ETH Zürich bei den Basisprüfungen nach dem ersten Studienjahr nicht nur Studierende des Schwerpunktfachs Physik und Anwendungen der Mathematik besonders gut ab, sondern auch solche mit dem gymnasialen Profil Latein/Griechisch (ETH, 2008). In einer deutschen Studie wiederum konnte für bestimmte Studienfächer nachgewiesen werden, dass Studierende, die Leistungskurse mit einem engen inhaltlichen Bezug zum späteren Studienfach belegt hatten, bessere Studienabschlussnoten erzielten als Abiturienten mit Leistungskursen ohne Bezug zu ihrem Studienfach (Fries, 2002). Ob sich ein ähnliches Ergebnis für die vorliegende Schweizer Stichprobe findet, ist Gegenstand der folgenden empirischen Untersuchung.

\section{Fragestellung}

Im vorliegenden Beitrag wird die Frage untersucht, ob die fachliche Nähe von gymnasialem Bildungsprofil und der gewählten Studienrichtung mit positiven Effekten auf Studienleistungen einhergeht. Dazu wird zuvor aus Sicht der Bildungsprofile dargestellt, welche Studienrichtungen jeweils etwa dreieinhalb Jahre später studiert werden.

\section{Me thode}

\section{Datengrundlage und Stichprobe}

Für den vorliegenden Beitrag werden Daten von Teilnehmenden der vom Schweizerischen Nationalfonds geförderten EVAMAR II-Nachfolge-Studie «Studierfähigkeit von Maturandinnen und Maturanden» verwendet. In dieser Studie wurde eine Deutschschweizer Teilstichprobe der EVAMAR II-Studie dreieinhalb, vier und fünf Jahre nach ihrer im Jahr 2007 abgelegten Matura zu ihrem Studium befragt. Die vorliegenden Analysen stützen sich auf Daten von insgesamt $\mathrm{N}=1203$ Studierenden (65\% weiblich), die sowohl an der postalisch durchgeführten sogenannten Statusbefragung im Winter 2010/2011 (Rücklaufquote ca. $60 \%$ ) als auch an der ein halbes Jahr später nachfolgenden Online-Befragung teilgenommen haben (Rücklaufquote ca. $75 \%$ ).

Der Anteil an Studierenden, die den Studiengang gewechselt haben, beträgt zum Zeitpunkt der Statuserhebung ca. 17 Prozent, was in etwa dem schweizerischen Durchschnitt entspricht (Diem, 2016). Die Variable des Studiengangs und der Indikatoren des Studienerfolgs beziehen sich damit im Fall eines Wechsels auf bereits gewechselte Studiengänge. Darüber hinaus gilt zu berücksichtigen, 
dass sich in der Stichprobe sehr wenige Studienabbrecher befinden: Während gesamtschweizerischen Zahlen zufolge der Anteil an Studienabbrechenden mit ca. 28 Prozent zu veranschlagen ist (Wolter, Diem \& Messer, 2013), liegt er in unserer Stichprobe bei lediglich rund 10 Prozent zum Zeitpunkt der Online-Befragung.

\section{Erhebungsinstrumente}

Bildungsprofil. Die Studierenden wurden in der Online-Erhebung gefragt, welches Schwerpunktfach (SPF) und welche Grundlagenfächer (GF) sie im Jahr der Matura belegt hatten. In Anlehnung an EVAMAR I orientieren wir uns für die Kategorisierung der individuellen Bildungsprofile ebenfalls am SPF (Ramseier et al., 2005). In einem zweiten Schritt wurde für eine Ausdifferenzierung der sprachlichen Bildungsprofile zusätzlich der Besuch des fremdsprachlichen Grundlagenfachs hinzugezogen, wodurch drei sprachliche Profile entstehen: Das altsprachliche Profil umfasst diejenigen Maturandinnen und Maturanden, die Latein und Griechisch als Schwerpunktfach (SPF) und als zweites fremdsprachliches Grundlagenfach (GF) gewählt haben; beim alt/neusprachlichen Profil haben die Inhaber eine alte und eine neue Sprache als SPF und GF gewählt; Jugendliche des neusprachlichen Profils haben zwei neue Sprachen als SPF und GF belegt. Die beiden Schwerpunktfächer Bildnerisches Gestalten und Musik wurden zu einem musischen Bildungsprofil zusammengefasst. Insgesamt wurden damit acht Bildungsprofile gebildet, denen jeweils verwandte Studienrichtungen zugeordnet wurden (vgl. Tabelle 1). Die Verteilung der Versuchspersonen unserer Stichprobe auf die einzelnen Bildungsprofile ist aus Tabelle 2 ersichtlich.

Studienrichtungen. Die Versuchspersonen wurden zu allen Messzeitpunkten nach ihren Studienfächern gefragt. Diese wurden auf Grundlage des «SHIS-Fächerkatalogs für Universitäre Hochschulen» sowie der «Offiziellen Klassierung der Studiengänge FH-PH», herausgegeben vom Bundesamt für Statistik (BfS), insgesamt 15 Kategorien zugeordnet und in einem weiteren Schritt zu zehn Kategorien zusammengefasst. Analysen auf der Basis von Zahlen des BfS zeigen, dass die Stichprobe zum Zeitpunkt der Statuserhebung - mit Ausnahme des überproportional vertretenen Anteils an Naturwissenschaftsstudierenden und des etwas geringeren Anteils an Studierenden des Bauwesens recht gut die gesamtschweizerische Verteilung widerspiegelt. Die Verteilung der Studierenden unserer Stichprobe auf die zusammengefassten zehn Studienrichtungen ist ebenfalls aus Tabelle 2 ersichtlich.

Inhaltliche Verwandtschaft von Schwerpunktfach und Studienfach. Die Charakterisierung der Kombinationen der Studienfachrichtungen mit den gymnasialen Bildungsprofilen als «verwandt» bzw. «nicht verwandt» erfolgte in Anlehnung an die bei der EVAMAR I-Studie vorgenommene Kategorisierung des «Standard-Übergangs» (Ramseier et al., 2005; ähnlich bei Notter \& Arnold, 2006). Danach handelt es sich um einen «Standard-Übergang», wenn Maturandinnen und Maturanden mit einem bestimmten Bildungsprofil sich 
im Mittel auf diese Studienrichtung ausserordentlich oft (zu über 85\%) oder weit öfter als die Gesamtheit gut oder eher gut vorbereitet fühlen oder wenn sie die Studienrichtung weit öfter wählen als andere (Ramseier et al., 2005). Die daraus resultierenden Kombinationen weisen nach unserer Einschätzung inhaltliche Ähnlichkeiten auf, so dass wir die Einteilung für die Untersuchung unserer Fragestellung adaptieren. Lediglich bei der Zuweisung des neusprachlichen Profils sehen wir keine inhaltliche Verwandtschaft zu den häufig von diesem Bildungsprofil gewählten sozialwissenschaftlichen Studienrichtungen, so dass wir dieses Profil nur mit den geistes- und sprachwissenschaftlichen Studienrichtungen als «verwandt» deklarieren. Die inhaltliche Ähnlichkeit ist für unsere Fragestellung von Bedeutung, da von einem Wissensvorsprung in dem jeweils verwandten Studienbereich ausgegangen wird. Es ergeben sich daraus folgende Kombinationen verwandter Bildungsprofile mit 15 Studienrichtungen:

\section{Tabelle 1: Vom Bildungsprofil zur Studienrichtung: verwandte Kombinationen}

\begin{tabular}{l|l} 
Gymnasiales Bildungsprofil & Verwandte Studienrichtung \\
\hline Altsprachlich & $\begin{array}{l}\text { Philosophie/Theologie; Sprach-/Literaturwis- } \\
\text { senschaften; Geschichte }\end{array}$ \\
\hline Alt-/Neusprachlich & Sprach-/Literaturwissenschaften; Geschichte \\
\hline Neusprachlich & Sprach-/Literaturwissenschaften \\
\hline Physik \& Anwendungen der Mathematik & $\begin{array}{l}\text { Mathematik/Informatik; Naturwissenschaften; } \\
\text { grüne und harte Ingenieurwissenschaften inkl. } \\
\text { Bauwesen }\end{array}$ \\
\hline Biologie \& Chemie & $\begin{array}{l}\text { Medizin; Naturwissenschaften; grüne } \\
\text { Ingenieurwissenschaften }\end{array}$ \\
\hline Wirtschaft \& Recht & Wirtschaftswissenschaften; Recht \\
\hline Philosophie, Pädagogik \& Psychologie & $\begin{array}{l}\text { Philosophie/Theologie; Sozialwissenschaften; } \\
\text { Lehrerbildung }\end{array}$ \\
\hline Musisch & Kunst-/Musikwissenschaften; Lehrerbildung \\
\hline
\end{tabular}

Die Studienrichtung der interdisziplinären Fächer (Sportwissenschaft und Ökologie) konnten keinem Bildungsprofil zugeordnet werden. Diese (zudem zahlenmässig kleine) Studienrichtung wird daher zum Teil von den Analysen ausgeschlossen.

Studienerfolg. Im vorliegenden Beitrag dienen Studienleistungen und Studiennoten als Indikatoren für die abhängige Variable. Alle Variablen gingen so in die Analysen ein, dass hohe Werte für hohen Studienerfolg stehen. Als Indikatoren für den Studienerfolg dienen folgende vier Skalen bzw. Items:

(1) Leistung im Vergleich: Bei dieser Variable aus der Onlinebefragung handelt es sich um eine Skala mit fünf Items, die aus der TOSCA-Studie (Trautwein et al., 2006) übernommen wurde. Ein Beispielitem lautet: «Verglichen mit anderen Studierenden bin ich im Studium sehr erfolgreich». Die Antwortmöglichkeiten 
reichten von $(1)=$ trifft überhaupt nicht zu bis $(4)=$ trifft völlig zu. Cronbachs Alpha beträgt .79.

(2) Durchschnittliche Note der Studienleistungen im aktuellen Studium. Studierende, die zum Zeitpunkt der zweiten Erhebung noch nicht über einen Bachelorabschluss verfügten, wurden gebeten, ihre bisherigen Studienleistungen auf einer vorgegebenen 6-stufigen Skala einzuschätzen $((1)=$ Noten 5.5-6.0, $(2)=$ 5.0-5.4, (3) = 4.5-4.9, (4) = 4.0-4.4, (5) = 3.5-3.9, (6) = Noten 3.0-3.4). Für die Mittelwertvergleiche wurde die Skala recodiert.

(3) Bachelornote. Studierende, die zum Zeitpunkt der Onlineerhebung bereits einen Bachelorabschluss vorweisen konnten, gaben bei dieser Variable ihre Gesamtbachelornote an (offene Frage). Von den Teilnehmenden wurde je nach Stand des Studiums alternativ die durchschnittliche Leistung im aktuellen Studium oder die Bachelornote erfragt.

(4) Anzahl Prüfungen, die nicht im 1. Anlauf bestanden wurden auf Bachelorstufe. Die Studierenden wurden gebeten, auf einer Skala von (1) = «0 Prüfungen oder Arbeiten nicht im ersten Anlauf bestanden» bis (5) = «> 3 Prüfungen oder Arbeiten nicht bestanden» Auskunft über die Zahl nicht bestandener Leistungsnachweise in ihrem abgeschlossenen Bachelorstudium zu geben. Für die Mittelwertvergleiche wurde die Skala recodiert.

Weiterhin werden zum Teil das Geschlecht und die Maturanote in den Analysen berücksichtigt. Die Maturanote wurde als Selbstauskunft offen erfragt.

\section{Ergebnisse}

\section{Verteilung der Studienrichtungen auf Bildungsprofile} Zunächst wurde der Frage nachgegangen, wie sich die hochschulischen Studienrichtungen auf die oben beschriebenen Bildungsprofile verteilen. In Tabelle 2 sind die Ergebnisse der entsprechenden Kreuztabelle dargestellt. Enthalten sind in den einzelnen Zellen, wieviel Prozent des jeweiligen Bildungsprofils die in den Zeilen aufgeführten Studienrichtungen studieren. Eine Schattierung einer Zelle signalisiert, dass die Prozentzahl von der erwarteten Prozentzahl, die sich bei einer Gleichverteilung ergäbe (= Angaben in der letzten Spalte), signifikant positiv oder negativ abweicht (Testgrösse: standardisiertes Residuum). Um nicht zu viele Zellen mit einer erwarteten Häufigkeit von weniger als fünf Personen zu erhalten, wurde die Kategorisierung mit den zehn Studienrichtungen gewählt.

Es lässt sich in Tabelle 2 ablesen, dass aus Sicht der Bildungsprofile insbesondere Maturandinnen und Maturanden mit einem mathematischen-physikalischen Schwerpunktfach dreieinhalb Jahre nach der Matur überzufällig häufig technische Wissenschaften (27.8\% im Vergleich zu den bei einer Gleichverteilung $\mathrm{zu}$ erwartenden $7.9 \%$ ) sowie mathematisch-naturwissenschaftliche Studienfächer studieren (33.3\% statt 13.8\%), jene mit SPF Biologie und Chemie sich besonders häufig für ein Medizinstudium (30.7\% statt $12.4 \%$ ) oder ein 
Bildungsprofil

\begin{tabular}{|c|c|c|c|c|c|c|c|c|c|}
\hline $\begin{array}{l}\text { Studienfach- } \\
\text { richtung }\end{array}$ & 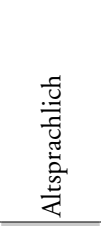 & 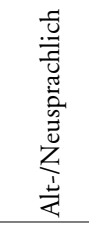 & 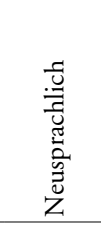 & 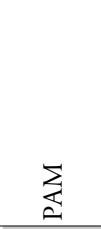 & $\underset{\mathscr{D}}{\cup}$ & $\begin{array}{l}\frac{1}{d} \\
\frac{X}{3}\end{array}$ & $\hat{\underline{\Xi}}$ & 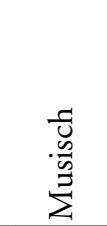 & 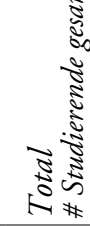 \\
\hline Geisteswiss. & $16.1 \%^{+}$ & $12.0 \%$ & $9.5 \%$ & $2.2 \%^{-}$ & $3.6 \% 0^{-}$ & $4.7 \%^{-}$ & $10.1 \%$ & $10.1 \%$ & $\begin{array}{c}8.6 \% \\
100\end{array}$ \\
\hline Wirtschaftswiss. & $13.7 \%$ & $13.6 \%$ & $12.7 \%$ & $16.7 \%$ & $9.5 \%$ & $32.7 \%^{+}$ & $5.8 \%^{-}$ & $5.8 \%^{-}$ & $\begin{array}{c}14.9 \% \\
173\end{array}$ \\
\hline Recht & $5.6 \%$ & $9.9 \%$ & $10.3 \%$ & $4.4 \%$ & $3.6 \% 0^{-}$ & $17.8 \%^{+}$ & $4.3 \%$ & $7.7 \%$ & $\begin{array}{c}9.1 \% \\
105\end{array}$ \\
\hline $\begin{array}{l}\text { Naturwiss. (inkl. } \\
\text { Mathe, Inf.) }\end{array}$ & $16.1 \%$ & $11.0 \%$ & $10.3 \%$ & $33.3 \% \%^{+}$ & $24.1 \%^{+}$ & $11.2 \%$ & $2.9 \% 0^{-}$ & $8.2 \%{ }^{-}$ & $\begin{array}{c}13.8 \% \\
160\end{array}$ \\
\hline $\begin{array}{l}\text { Medizin und } \\
\text { Pharmazie }\end{array}$ & $16.1 \%$ & $15.7 \%$ & $6.3 \%$ & $6.7 \%$ & $30.7 \% \%^{+}$ & $6.1 \%-$ & $8.7 \%$ & $9.2 \%$ & $\begin{array}{c}12.4 \% \\
144\end{array}$ \\
\hline Techn. Wiss. & $6.5 \%$ & $6.8 \%$ & $1.6 \%-$ & $27.8 \% \%^{+}$ & $15.3 \%^{+}$ & $6.5 \%$ & $1.4 \%$ & $3.9 \% 0^{-}$ & $\begin{array}{c}7.9 \% \\
92\end{array}$ \\
\hline Interdisziplinäre & $2.4 \%$ & $3.1 \%$ & $3.2 \%$ & $2.2 \%$ & $4.4 \%$ & $0.9 \%$ & $2.9 \%$ & $1.9 \%$ & $\begin{array}{c}2.2 \% \\
34\end{array}$ \\
\hline Lehrerbildung & $7.3 \%$ & $8.9 \%$ & $18.3 \%^{+}$ & $2.2^{-}$ & $2.9^{-} 0^{-}$ & $7.5 \%$ & $26.1 \%^{+}$ & $20.3 \%^{+}$ & $\begin{array}{c}11.3 \% \\
131\end{array}$ \\
\hline Musik und Kunst & $2.4 \%$ & $2.6 \%$ & $0.8 \%$ & $0.0 \%$ & $0.7 \%$ & $0.9 \%$ & $0.0 \%$ & $11.6 \% 0^{+}$ & $\begin{array}{c}3.1 \% \\
36\end{array}$ \\
\hline Sozialwiss. & $13.7 \%$ & $16.2 \%$ & $27.0 \% \%^{+}$ & $4.4 \%^{-}$ & $5.1 \%^{-}$ & $11.7 \%$ & $37.7 \%^{+}$ & $21.3 \%$ & $\begin{array}{c}16.2 \% \\
188\end{array}$ \\
\hline Total & $100 \%$ & $100 \%$ & $100 \%$ & $100 \%$ & $100 \%$ & $100 \%$ & $100 \%$ & $100 \%$ & $100 \%$ \\
\hline $\begin{array}{l}\text { \# Studierende } \\
\text { gesamt }\end{array}$ & 124 & 191 & 126 & 90 & 137 & 214 & 69 & 207 & 1158 \\
\hline
\end{tabular}

Schattiert: Prozentwert des Bildungsprofils weicht signifikant vom Prozentwert aller Befragten ab (Spalte «Total»; standardisiertes Residuum < -2 bzw. > 2); Lesebeispiel erste Zelle: $16.1 \%$ des altsprachlichen Bildungsprofils studieren ein geisteswissenschaftliches Studienfach; ${ }^{+}=$überzufällig häufige Wahl, - = überzufällig seltene Wahl; PAM $=$ Physik und Anwendungen der Mathematik, B\&C = Biologie und Chemie, W\&R= Wirtschaft und Recht, PPP = Philosophie/Psychologie/Pädagogik

naturwissenschaftliches Studium entschieden haben (24.1\% statt 13.8\%), Personen des Bildungsprofils Wirtschaft und Recht die entsprechend korrespondierenden Studienrichtungen Wirtschaftswissenschaften (32.7\% statt 14.9\%) und Rechtswissenschaften (17.8\% statt 9.1\%) absolvieren sowie ehemalige Maturan- 
dinnen und Maturanden mit einem Schwerpunktfach PPP signifikant häufig ein sozialwissenschaftliches Studium (37.7\% statt 16.2\%) oder ein Lehrerbildungsstudium $(26.1 \%$ statt $11.3 \%)$ ergriffen haben. Personen mit einem musischen Profil studieren ca. dreieinhalb Jahre später überzufällig häufig ein Studium der Musik oder Kunst (11.6\% statt 3.1\%) oder absolvieren ein Lehrerbildungsstudium (20.3\% statt $11.3 \%)$. Schülerinnen und Schüler mit neusprachlichem Profil haben sich signifikant überzufällig für Studiengänge der Sozialwissenschaften eingeschrieben (27.0\% statt 16.2\%). Lediglich für das alt/neusprachliche Profil können keine signifikanten Präferenzen für bestimmte Studienrichtungen festgestellt werden. Die Ergebnisse verdeutlichen mit einem Kontingenzkoeffizienten von .54, dass die Koppelungen nicht unabhängig von den Bildungsprofilen sind $\left(\mathrm{Chi}^{2}=459.172, \mathrm{df}=56, \mathrm{p}<.001\right.$ bei Weglassen der Studienrichtung der quantitativ zu kleinen interdisziplinären Studienrichtungen).

\section{Deskriptive Ergebnisse zur Variable "Verwandtschaft" der Bildungsprofil-Studienrichtungen-Kombinationen}

Dreieinhalb Jahre nach der Matura belegen 33.7\% der vorliegenden Stichprobe ein Studienfach, das nach obiger Zuordnung inhaltlich mit ihrem Schwerpunktfach verwandt ist. Dabei lassen sich für die Bildungsprofile unterschiedlich stark ausgeprägte Koppelungen feststellen $\left(\mathrm{Chi}^{2}=221.199, \mathrm{df}=7, \mathrm{p}\right.$ $<.001$, Kontingenzkoeffizient $=.394)$, die bereits in Tabelle 2 angedeutet sind: Während die drei sprachlichen Bildungsprofile im Vergleich zur Gesamtstichprobe signifikant häufiger eher nicht Fächer studieren, die dem Bildungsprofil fachlich verwandt sind, studieren Personen der MINT-Bildungsprofile PAM und $\mathrm{B} \& \mathrm{C}$ sowie von Wirtschaft \& Recht und PPP überzufällig häufig Fächer, die ihrem Bildungsprofil inhaltlich ähnlich sind. Für die musischen Profile lässt sich keine Tendenz erkennen. Weiterhin lässt sich festhalten, dass Studenten häufiger ein verwandtes Studienfach belegen als Studentinnen $\left(\mathrm{Chi}^{2}=19.933\right.$, $\mathrm{df}=1, \mathrm{p}<.001)$. Im nächsten Schritt wird der Frage nachgegangen, ob die inhaltliche Verwandtschaft Effekte auf Studienleistungen zeigt.

\section{Schwerpunktfach-Studienfach-Koppelung und Studienerfolg}

Noten unterliegen sehr unterschiedlichen Bewertungsmassstäben und können damit auch von der Studienrichtung abhängen (Schiefele, Streblow, Ermgassen \& Moschner, 2003). Daher wurde zur Beantwortung der Forschungsfrage, ob sich Unterschiede in den Studienleistungen der beiden Gruppen ergeben (Faktor «Verwandtschaft»), für alle Indikatoren des Studienerfolgs allgemeine lineare Modelle unter Kontrolle des Faktors der Studienfachrichtung gerechnet. Weiterhin wurden zur Kontrolle das Geschlecht sowie die Maturanote als Kovariaten in die Modelle aufgenommen. In Tabelle 3 sind die deskriptiven Ergebnisse der entsprechenden Varianzanalysen des Haupteffekts des Faktors «Verwandtschaft» sowie die F-Werte aller Haupt- und Interaktionseffekte dargestellt. 
Tabelle 3: Ergebnisse allgemeiner linearer Modelle mit den Faktoren "Verwandtschaft», "Studienrichtung" und den Kovariaten "Geschlecht» und "Maturanote" sowie den Interaktionstermen "Verwandtschaft *Studienrichtung", "Verwandtschaft ${ }^{*}$ Geschlecht», "Verwandtschaft ${ }^{*}$ Maturanote; Mittelwerte, Standardabweichung, F-Werte und p-Werte der Effekte

\begin{tabular}{lcccccccccccccc} 
& \multicolumn{2}{c}{$\begin{array}{c}\text { Leistung im } \\
\text { Vergleich }\end{array}$} & \multicolumn{4}{c}{$\begin{array}{c}\text { Aktuelle } \\
\text { BA-Leistung }\end{array}$} & \multicolumn{4}{c}{$\begin{array}{c}\text { BA- } \\
\text { Abschlussnote }\end{array}$} & \multicolumn{4}{c}{$\begin{array}{c}\text { Anzahl durchge- } \\
\text { fallener Prüfungen }\end{array}$} \\
\hline $\begin{array}{lccccccccccccccc}\text { Fachliche } \\
\text { Verwandtschaft }\end{array}$ & $\mathrm{N}$ & $\mathrm{M}$ & $\mathrm{SD}$ & $\mathrm{N}$ & $\mathrm{M}$ & $\mathrm{SD}$ & $\mathrm{N}$ & $\mathrm{M}$ & $\mathrm{SD}$ & $\mathrm{N}$ & $\mathrm{M}$ & $\mathrm{SD}$ \\
$\quad$ Nein & 692 & 2.86 & .53 & 348 & 4.36 & .85 & 328 & 5.06 & .36 & 351 & 4.21 & 1.21 \\
Ja & 381 & 2.88 & .53 & 159 & 4.39 & .87 & 208 & 5.03 & .34 & 231 & 4.23 & 1.14 \\
Gesamt & 1073 & 2.87 & .53 & 507 & 4.36 & .86 & 536 & 5.05 & .35 & 582 & 4.22 & 1.18
\end{tabular}

Haupteffekte

Verwandtschaft

F-Wert/p-Wert $.186 / .667$

$.208 / .649$

$.195 / .659$

$.045 / .832$

$\mathrm{Eta}^{2}$

.000

.001

.001

.000

Studienrichtung

F-Wert/p-Wert

$3.247 / .001$

$4.848 /<.001$

$8.787 /<.001$

$3.129 / .002$

$\mathrm{Eta}^{2}$

.034

.106

.161

.060

Geschlecht

F-Wert/p-Wert

$.817 / .366$
.001

$.706 / .401$

$1.500 / .221$

.004

1.734/.189

.002

.004

Maturanote

F-Wert/p-Wert

$59.380 /<.001$

$63.535 /<.001$

.162

$71.142 /<.001$

.162

$30.798 /<.001$

.075

162

.073

Interaktionseffekte

Verwandtschaft*

Studienrichtung

F-Wert/p-Wert

$\mathrm{Eta}^{2}$

$.992 / .441$

.011

Verwandtschaft*

Geschlecht

F-Wert/p-Wert

$\mathrm{Eta}^{2}$

Verwandtschaft*

Maturanote

F-Wert/p-Wert

$\mathrm{Eta}^{2}$

$.150 / .699$

$.219 / .640$

$.338 / .561$

$.078 / .780$

.000
$.490 / .484$

.001

$.886 / .347$

.002
$1.890 / .060$

040

.030

Die Interaktionseffekte der Verwandtschaftsvariablen wurden modelliert, um der Frage nachgehen zu können, ob sich eventuelle Abhängigkeiten der Studien- 
erfolgskriterien von der Gruppenzugehörigkeit für verschiedene Studienrichtungen sowie abhängig vom Geschlecht und der Maturanote unterschiedlich darstellen. Die Studierenden der «Interdisziplinären Studienrichtung» wurden von diesen Analysen wiederum ausgeschlossen.

Tabelle 3 verdeutlicht, dass sich die Mittelwerte aller Studienerfolgsindikatoren für die beiden Gruppen kaum unterscheiden. Demnach spielt es für das Ausmass der hier betrachteten Studienerfolgskriterien keine Rolle, ob die BA-Absolventinnen und -absolventen bzw. die Studierenden ein Schwerpunktfach im Gymnasium belegt hatten, das eine inhaltliche Ähnlichkeit zu ihrem Studienfach aufweist oder nicht. Als entsprechend niedrig erweisen sich die nicht signifikanten F-Werte der Haupteffekte "Verwandtschaft» unter Kontrolle der Effekte des Geschlechts, der Studienrichtung und der Maturanote. Wie erwartet zeigen sich sowohl für die Maturanote als auch für die Studienrichtungen signifikante Effekte. Studentinnen und Studenten unterscheiden sich dagegen nicht im hier erhobenen Studienerfolg. Das Fehlen signifikanter Interaktionseffekte zeigt an, dass die Irrelevanz der Schwerpunktfachaffinität für das Ausmass des Studienerfolgs im Durchschnitt über alle Studienrichtungen hinweg und unabhängig vom Geschlecht und der Maturanote gesehen gilt.

\section{Diskussion}

In vorliegendem Beitrag wurde zum ersten Mal für die Schweiz systematisch untersucht, ob die Gruppe von Studierenden, die eine Fachrichtung eingeschlagen haben, die mit dem von ihnen besuchten gymnasialen Bildungsprofil inhaltliche Ähnlichkeiten aufweist, mehr Studienerfolg hat als die Gruppe Studierender, die eine Studienrichtung gewählt haben, die nicht fachlich mit ihrem Bildungsprofil verwandt ist. Es zeigten sich keine Unterschiede im Ausmass der Studienleistungen zwischen den beiden Gruppen. Der Blick auf die Kombinationen von Bildungsprofil und Studienfächern ergab eine insgesamt eher lockere Koppelung von schulischer und hochschulischer Ausbildung, bei gleichzeitig engen Beziehungen einzelner Schwerpunktfächer zu verwandten Studienrichtungen. Im Folgenden werden die Ergebnisse im Hinblick auf das im Theorieteil beschriebene Bildungsziel der allgemeinen Studierfähigkeit diskutiert.

Mit dem Ziel verbindet sich der Anspruch, dass jede Maturandin und jeder Maturand am Ende des Gymnasiums in der Lage sein sollte, jedes beliebige Fach an einer Schweizer Hochschule studieren zu können. Dies impliziert, dass zum einen die Studienfachwahl für alle unbeschränkt möglich sein sollte und des Weiteren, dass alle genügend - subjektiv wahrgenommen und objektiv gesehen - kompetent sein sollten, um jede Studienrichtung wählen und studieren zu können.

Im Hinblick auf eine freie Studienfachwahl wird eine enge Koppelung von Studienfachwahlen an verwandte Bildungsprofile zum Teil kritisch gesehen, 
da damit die Gefahr bestünde, dass die Studienfachwahl bereits auf Ebene der Sekundarstufe massgeblich vorgespurt oder sogar eingeschränkt würde (Notter \& Arnold, 2006; Ramseier et al., 2005). Der vorliegende Befund einer insgesamt eher lockeren Koppelung, der den Ergebnissen der EVAMAR I-Untersuchung ähnelt (Ramseier et al., 2005), spricht grundsätzlich für die Offenheit der Studienfachwahlen bis zur Matur. Allerdings weisen unsere Daten im Einklang mit verschiedenen Studien (z. B. Berweger et al., 2015; Nagy et al., 2006; SKBF, 2014) darauf hin, dass die Wahl von MINT-Studienfächern häufig bereits früh vorgespurt wird und die Studienrichtungen der Naturwissenschaften mit Mathematik und Informatik sowie die Technischen Wissenschaften ihre Studierenden vor allem aus den verwandten Schwerpunktfächern PAM und B\&C rekrutieren. Dies kann man angesichts der weltweit hohen Nachfrage nach qualifizierten Arbeitskräften in diesen Bereichen bei gleichzeitig einer als zu gering eingeschätzten Anzahl MINT-Studierender (OECD, 2008) durchaus als kritisch ansehen, zumal PAM nicht sehr häufig und zusätzlich von nur wenigen Frauen gewählt wird (BFS, 2016). Soll die bildungspolitisch gewünschte Forcierung der Nachfrage nach MINT-Studiengängen unterstützt werden, unterstreicht der Befund, dass bereits vor der Schwerpunktfachwahl das Interesse an MINT-Fächern geweckt werden muss (Volodina et al., 2015). Wie oben diskutiert, entspräche diese frühe Spezialisierung allerdings nicht dem Ziel der allgemeinen Studierfähigkeit. Eine andere Möglichkeit wäre, Maturandinnen und Maturanden anderer Schwerpunktfächer stärker das Vertrauen zu vermitteln, dass sie sich aufgrund guten MINT-Vorwissens kompetent genug fühlen, entsprechende Studienfächer auch ohne vorherige Vertiefung im Gymnasium bewältigen zu können.

Bezüglich objektiv festgestellter Kompetenzen wurden in der Studie EVAMAR II für die Gesamtstichprobe, aus der die Stichprobe der vorliegenden Untersuchung rekrutiert wurde, Unterschiede im Hinblick auf studierrelevantes Wissen zwischen verschiedenen Schwerpunktfachgruppen kurz vor der Maturitätsprüfung konstatiert (Eberle et al., 2008). Beispielsweise schnitten die PPP-Absolventinnen und -Absolventen und jene des musischen Profils im Durchschnitt in den Mathematikleistungstests signifikant schlechter ab als die anderen Profile. Ein Übergang in mathematiklastige, und damit überwiegend schwerpunktfachfremde Studiengänge bietet sich für Absolventinnen und Absolventen dieser Bildungsprofile daher auch aus subjektiver Sicht wenig an - was jedoch der Idee der allgemeinen Studierfähigkeit widerspricht. Andererseits zeigen unsere Ergebnisse bezüglich der mangelnden Unterschiede beim Studienerfolg, dass entweder Lücken und Wissensdefizite bis zum Studienabschluss nicht so gravierend ausfallen oder kompensiert werden, was wiederum für ein Gelingen der Sicherung der allgemeinen Studierfähigkeit spricht.

Allerdings gilt dieser Zusammenhang nur für jene Studierenden, die das Studienziel des BA-Abschlusses auch tatsächlich erreicht haben bzw. auf dem besten Weg dorthin sind - was für alle Personen unserer Stichprobe aufgrund 
der analysierten Studienerfolgskriterien zutrifft.

In der Operationalisierung des Studienerfolgs mit der Konzentration auf die Abschlussnoten bzw. die Studienleistungen ist sicherlich eine Limitation der vorliegenden Studie zu sehen. Dies betrifft weniger die Problematik, dass diese Leistungen per Selbstauskunft erhoben wurden - Metastudien zeigen, dass selbstberichtete Durchschnittsnoten sehr hoch mit offiziellen Angaben korrelieren $(\mathrm{r}=$.90) (Kuncel, Credé \& Thomas, 2005). Wäre jedoch eine Betrachtung von Studienabbruch als Indikator für Studienerfolg im Fokus gestanden, hätten sich möglicherweise durchaus Unterschiede zwischen den beiden Gruppen gezeigt. Dies legen die im Theorieteil berichteten Befunde zur Bedeutung des Vorwissens für den Erfolg zu Beginn des Studiums sowie zum Verbleib im Studium insbesondere für naturwissenschaftliche und ingenieurwissenschaftliche Studiengänge nahe. Zudem hatten einer weiteren Studie zufolge Personen, die ein mit dem Studienfach verwandtes Maturitätsprofil aufwiesen, ein geringeres Wechselrisiko als Personen, die ein mit dem Studienfach wenig verwandtes Maturitätsprofil besucht hatten (Diem, 2016; ähnlich bei Notter \& Arnold, 2006). Im Verbund mit den Hinweisen auf einen positiven Zusammenhang zwischen Studienwechseln und der Wahrscheinlichkeit eines Studienabbruchs (Diem, 2016; Wolter et al., 2013), lässt sich vermuten, dass Studienabbrecher eher aus der Gruppe der Studierenden mit einem bildungsprofilfremden Studienfach stammen. Vor diesem Hintergrund ist denkbar, dass von dieser Gruppe eher die leistungsstärkeren Personen in unserer Stichprobe vertreten sind, während eher Leistungsschwache dieser Gruppe das Studium mittlerweile abgebrochen haben könnten und daher in unserer Stichprobe fehlen oder aber in einen ihrem Schwerpunktfach ähnlichen Fachbereich gewechselt haben, was Spiess (1997) zufolge einer typischen Wechselrichtung entspräche. Dies könnte ein Grund dafür sein, dass sich keine Unterschiede beim Studienerfolg ergeben haben. Zukünftige Studien sollten daher in näheren Abständen zur Matura als in der vorliegenden Studie realisiert, längsschnittliche Erhebungen im Zeitablauf des Studiums unter dem Aspekt von Studienfachwechseln und Studienabbrüchen durchführen. Zugleich wäre die Frage zu stellen, ob eine Rückkehr zum Konzept der Fakultätsreife mit einem höheren Grad an Spezialisierung und den damit verbundenen Vorteilen spezialisierten Vorwissens nicht zu geringeren Studienabbruchszahlen führen würde.

Weitere Grenzen der vorliegenden Studie sind darin zu sehen, dass das Ergebnis bezüglich der Unterschiede beim Studienerfolg von der Definition der "Verwandtschaftsvariablen» abhängt. Die Zuweisungen der verschiedenen Bildungsprofile zu den Studienfächern als "verwandt» sind jedoch nicht für alle Bildungsprofile und alle Paare von Schul- und Studienfächern gleichermassen eindeutig zu bestimmen (Ramseier et al., 2005). Eine andere Einteilung hätte daher möglicherweise zu anderen Ergebnissen in unserer Studie geführt.

Schliesslich bleibt noch der Vergleich zur Studie von Fries (2002), in der deutsche Studierende mit studienfachnahen Leistungskursen bessere Studien- 
noten erzielten als solche, die einen studienfachfernen Leistungskurs besucht hatten. Ein Grund für dieses im Vergleich zur eigenen Untersuchung anders lautende Ergebnis könnte neben der bereits diskutierten Selektivität unserer Stichprobe darin liegen, dass die Schwerpunktfächer in der Schweiz quantitativ nicht den gleichen Umfang und qualitativ nicht die gleiche Bedeutung wie die deutschen Leistungskurse einnehmen. So gilt es die Besonderheit des Schweizer Gymnasiums zu berücksichtigen, die in dem international vergleichsweise sehr breit angelegten Kanon an Pflichtfächern in der Oberstufe besteht (Eberle \& Brüggenbrock, 2013), und der das zweite zentrale Bildungsziel der breiten Allgemeinbildung sichern hilft. Dieser breite Kanon steht einer im Rahmen dieser Diskussion zum Teil als vorteilhaft genannten verstärkten Spezialisierung entgegen. Die vorliegenden Befunde deuten darauf hin, dass der Kanon die angezielte "breit gefächerte, ausgewogene und kohärente Bildung» (MAR 95, Artikel 5) recht gut zu sichern vermag und damit auch die allgemeine Studierfähigkeit - zumindest für jene, die ihr Studium erfolgreich abzuschliessen vermögen.

\section{Anmerkung}

1 Die meisten Schulen oder Kantone bieten nicht alle Schwerpunktfächer an, ein durchschnittliches Angebot in der Deutschschweiz ermöglicht die Wahl zwischen sieben Schwerpunktfächern (Eberle \& Brüggenbrock, 2013, S. 45). Beispielsweise wurde das Schwerpunktfach «Philosophie, Pädagogik und Psychologie» schweizweit nur an 36 der 129 Gymnasien angeboten; es kann in dem in unserer Stichprobe sehr stark vertretenen Kanton Zürich mangels Angebot nicht belegt werden (Stand 2011, ebenda, S. 46).

\section{Literaturverzeichnis}

Abel, J. (2002). Kurswahl aus Interesse? Wahlmotive in der gymnasialen Oberstufe und Studienwahl. Die Deutsche Schule, 94, (2), 192-203.

Aeschlimann, B., Herzog, W. \& Makarowa, E. (2015). Frauen in MINT-Berufen: Retrospektive Wahrnehmung des mathematisch-naturwissenschaftlichen Unterrichts auf der Sekundarstufe I. Zeitschrift für Bildungsforschung, 5, (1), 37-49.

Alters, B. J. (1995). Counseling physics students: A research basis. The physics teacher, 33, 413-415.

Baron-Boldt, J., Schuler, H., \& Funke, U. (1988). Prädiktive Validität von Schulabschlußnoten: Eine Metaanalyse. Zeitschrift für Pädagogische Psychologie, 2, 79-90.

Berweger, S., Bieri Buschor, C. \& Keck Frei, A. (2015). Studienwahl MINT. Gymnasiastinnen aus mathematisch-naturwissenschaftlichen Bildungsprofilen und ihr Wunsch, Wissenschaftlerin zu werden. Psychologie in Erziehung und Unterricht, 62, 121-135.

Bundesamt für Statistik [BFS]. (2009). Studienfachwahl und Hochschulwahl. Motivationale Aspekte. Neuchâtel: BFS.

Bundesamt für Statistik [BFS]. (Hrsg.). (2016). Bildungsabschlüsse. Ausgabe 2016. Neuchâtel: BFS.

Burton, N. W. \& Ramist, L. (2001). Predicting success in college: SAT studies of classes graduating since 1980 (The College Board research report, 2001-2). New York City, NY: College Entrance Examination Board. 
Brüggenbrock, C., Eberle, F. \& Oelkers, J. (2016). Die jüngeren Entwicklungen des Gymnasiums und der Matura in der Schweiz. In J. Kramer, M. Neumann \& U. Trautwein (Hrsg.), Abitur und Matura im Wandel (S. 59-80). Wiesbaden: Springer VS.

Deidesheimer Kreis. (1997). Hochschulzulassung und Studieneignungstests. Göttingen: Vandenhoeck \& Ruprecht.

Diem, A. (2016). Studienfachwechsel im Bologna-System. Eine Analyse der universitären Hochschulen der Schweiz (SKBF Staff Paper 17). Aarau: SKBF.

Eberle, F. \& Brüggenbrock, C. (2013). Bildung am Gymnasium (EDK-Schriftenreihe «Studien + Berichte»-35A). Bern: EDK.

Eberle, F., Gehrer, K., Jaggi, B., Kottonau, J., Oepke, M. \& Pflüger, M. (2008). Evaluation der Maturitätsreform 1995. Schlussbericht zur Phase II. Bern: Staatssekretariat für Bildung und Forschung SBF.

Eccles, J. S. (1994). Understanding women's educational and occupational choices: Applying the Eccles et al. model of achievement-related choices. Psychology of women quarterly, 18, 585-609.

Eglin-Chappuis, N. (2007). Studienfachwahl und Fächerwechsel. Eine Untersuchung des Wahlprozesses im Übergang vom Gymnasium an die Hochschule. Bern: CEST.

ETH (2008). Maturanoten und Studienerfolg. Eine Analyse des Zusammenhangs zwischen Maturanoten und der Basisprüfung an der ETH Zürich. Zürich: ETH.

Freyer, K. (2013). Zum Einfluss von Studieneingangsvoraussetzungen auf den Studienerfolg Erstsemesterstudierender im Fach Chemie. Dissertation, Fakultät für Chemie der Universität Duisburg-Essen.

Fries, M. (2002). Abitur und Studienerfolg. Beiträge zur Hochschulforschung, 24, (1), 30-51.

Gottfredson, L. S. (1981). Circumscription and compromise: A developmental theory of occupational aspirations. Journal of counseling psychology monograph, 28, 545-579.

Hell, B., Trapmann, S. \& Schuler, H. (2007). Eine Metaanalyse der Prognosekraft von Studierfähigkeitstests. Empirische Pädagogik, 21, 251-270.

Heublein, U., Hutzsch, C., Schreiber, J., Sommer, D. \& Besuch, G. (2009). Ursachen des Studienabbruchs in Bachelor- und in herkömmlichen Studiengängen (HIS-Projektbericht). HIS \& Bundesministerium für Bildung und Forschung.

Kuncel, N. R., Credé, M. \& Thomas, L. L. (2005). The validity of self-reported grade point averages, class ranks, and test scores: A meta-analysis and review of the literature. Review of educational research, 75, 63-82.

Kuncel, N. R. \& Hezlett, S. A. (2007). Standardized tests predict graduate students' success. Science, 315, 1080-1081.

Lent, R. W., Brown, S. D. \& Hackett, G. (1994). Toward a unifying social cognitive theory of career and academic interest, choice, and performance. Journal of vocational behavior, 45, 79-122.

Linn, R. L. \& Hastings, C. N. (1984). A meta-analysis of the validity of predictor of performance in law school. Journal of educational measurement, 21, 245-259.

MAR. (1995). Verordnung des Bundesrates/Reglement der EDK über die Anerkennung von gymnasialen Maturitätsausweisen (MAR) vom 16. Januar/15. Februar 1995.

Nagy, G. (2005). Berufliche Interessen, kognitive und fachgebundene Kompetenzen: Ibre Bedeutung für die Studienfachwahl und die Bewährung im Studium. Dissertation, Universität Berlin, Berlin.

Nagy, G., Trautwein, U., Baumert, J., Köller, O. \& Garrett, J. (2006). Gender and course selection in upper secondary education: Effects of academic self-concept and intrinsic value. Educational research and evaluation, 12, 323-345.

Notter, P. \& Arnold, C. (2006). Der Übergang ins Studium II. Bericht zu einem Projekt der Konferenz der Schweizerischen Gymnasialrektoren (KSGR) und der Rektorenkonferenz der Schweizer Universitäten (CRUS). Bern: Staatssekretariat für Bildung und Forschung. 
OECD. (2008). Encouraging student interest in science and technology studies. Paris: OECD.

Oepke, M. \& Eberle, F. (2014). Studierfähigkeit und Studienfachwahl von Maturandinnen und Maturanden. In F. Eberle, B. Schneider-Taylor \& D. Bosse (Hrsg.), Abitur und Matura zwischen Hochschulvorbereitung und Berufsorientierung (S. 185-214). Wiesbaden: Springer.

Oepke, M. \& Eberle, F. (2016). Deutsch- und Mathematikkompetenzen - wichtig für die (allgemeine) Studierfähigkeit? In J. Kramer, M. Neumann \& U. Trautwein (Hrsg.), Abitur und Matura im Wandel (S. 215-252). Wiesbaden: Springer VS.

Ramist, L., Lewis, C. \& McCamley-Jenkins, L. (2001). Using achievement tests/SAT II: Subject Tests to demonstrate achievement and predict college grades: Sex, language, ethnic, and parental education groups (College Board Research Report No. 2001-5). New York, NY: College Entrance Examination Board.

Ramseier, E., Allraum, J., Stalder, U., Grin, F., Alliata, R., Müller, S., et al. (2005). Evaluation der Maturitätsreform 1995 (EVAMAR). Neue Fächerstruktur - Pädagogische Ziele - Schulentwicklung. Schlussbericht zur Phase 1. Bern: Schweizerische Konferenz der Erziehungsdirektoren und Bundesamt für Bildung und Wissenschaft.

Robbins, S. B., Lauver, K., Le, H., Davis, D., Langley, R. \& Carlstrom, A. (2004). Do psychosocial and study skill factors predict college outcomes? A meta-analysis. Psychological bulletin, 130, 261-288.

Rode Larsen, M., Bjørnøy Sommersel, H. \& Søgaard Larsen, M. (2013). Evidence on Dropout Phenomena at Universities. Copenhagen: Danish Clearinghouse for Educational Research, Aarhus University.

Sadler, P. M. \& Tai, R. H. (2001). Success in introductory college physics: the role of high school preparation. Science Education, 85, 111-136.

Schiefele, U., Streblow, L., Ermgassen, U. \& Moschner, B. (2003). Lernmotivation und Lernstrategien als Bedingungen der Studienleistung: Ergebnisse einer Längsschnittstudie. Zeitschrift für Pädagogische Psychologie, 17, 185-198.

Schnabel, K. U. \& Gruehn, S. (2000). Studienwünsche und Berufsorientierungen in der gymnasialen Oberstufe. In J. Baumert, W. Bos \& R. Lehmann (Hrsg.), TIMSS/III. Dritte internationale Mathematik- und Naturwissenschaftsstudie - Mathematische und naturwissenschaftliche Bildung am Ende der Schullaufbahn, Bd. 2. (S. 405-453). Opladen: Leske + Budrich.

Schumann, S., Eberle, F. \& Oepke, M. (2013). Ökonomisches Wissen und Können am Ende der Sekundarstufe II: Effekte der Bildungsgang-, Klassen- und Geschlechtszugehörigkeit. In U. Faßhauer, B. Fürstenau \& E. Wuttke (Hrsg.), Jahrbuch der berufs- und wirtschaftspädagogischen Forschung 2013 (S. 35-46). Opladen: Barbara Budrich.

Schwartz, M. S., Sadler, P. M., Sonnert, G. \& Tai, R. H. (2008). Depth versus breadth: How content coverage in high school science courses relates to later success in college science coursework. Science education, 93, 798-826.

Schweizerische Koordinationsstelle für Bildungsforschung [SKBF]. (2014). Bildungsbericht Schweiz 2014. Aarau: SKBF.

Spiess, C. (1997). Studienwechsel: Ausmass, Bedingungen und Folgen. Zürich: Rüegger.

Tai, R. H., Sadler, P. M., \& Loehr, J. F. (2005). Factors influencing success in introductory college chemistry. Journal of research in science teaching, 42, 987-1012.

Trapmann, S. (2008). Mehrdimensionale Studienerfolgsprognose: Die Bedeutung kognitiver, temperamentsbedingter und motivationaler Prädiktoren für verschiedene Kriterien des Studienerfolgs. Dissertation, Universität Hohenheim, Stuttgart.

Trapmann, S., Hell, B., Hirn, J. O. W. \& Schuler, H. (2007). Meta-analysis of the relationship between the Big Five and academic success at university. Zeitschrift für Psychologie, 215, $132-151$.

Trautwein, U., Husemann, N., Lüdtke, O., Köller, O., Watermann, R., Maaz, K., Nagy, G. \& Baumert, J. (2006). Transformation des Sekundarschulsystems und akademische 
Karrieren (TOSCA). Dokumentation der eingesetzten Items und Skalen. Welle 2. Berlin: Max-Planck-Institut für Bildungsforschung.

Volodina, A., Nagy, G. \& Retelsdorf, J. (2015). Berufliche Interessen und der Übergang in die gymnasiale Profiloberstufe: Ihre Struktur und Vorhersagekraft für das individuelle Wahlverhalten. Zeitschrift für Pädagogische Psychologie, 29, (2), 89-100.

Wolter, S. C., Diem, A. \& Messer, D. (2013). Studienabbrüche an Schweizer Universitäten. (SKBF Staff Paper 11). Aarau: SKBF.

Zwick, M. \& Renn, O. (2000). Die Attraktivität von technischen und ingenieurwissenschaftlichen Fächern bei der Studien- und Berufswahl junger Frauen und Männer. Stuttgart: Akademie für Technologiefolgenabschätzung.

Schlagworte: Studienerfolg, Übergang Gymnasium-Hochschule, Maturität, allgemeine Studierfähigkeit, Hochschulreife, Studienfachwahl, Schwerpunktfachwahl

\section{D'une option spécifique au gymnase aux études universitaires: le choix d'une filière académique apparentée à l'option spécifique suscite-t-il plus de réussite que celui d'une autre filière?}

\section{Ré sumé}

Le choix d'une option spécifique au gymnase est censé amener à une meilleure préparation aux études dans une filière voisine. L'objectif de cette recherche, menée sur un échantillon d'environ 1100 étudiants suisses alémaniques, était de mesurer si la proximité entre option spécifique suivie au gymnase et discipline académique mène à de meilleurs résultats à l'université. Les résultats montrent qu'en moyenne, la performance des personnes ayant choisi une discipline académique proche de leur option spécifique ne s'avère pas meilleure que celle des étudiants provenant d'une autre filière gymnasiale. Ces résultats sont discutés à la lueur de l'objectif principal de l'enseignement gymnasial, à savoir l'aptitude générale aux études.

Mots-clés: Réussite académique, transition du gymnase aux hautes écoles, maturité, aptitude générale aux études supérieures, choix des études, choix de l'option spécifique 


\section{Dall'opzione specifica liceale alla materia di studio all'università: Più successo nello studio tramite una materia affine piuttosto che una non affine all'opzione specifica?}

\section{Riassunto}

La scelta dell'opzione specifica liceale dovrebbe portare ad una preparazione approfondita per indirizzi di studio affini all'università. L'obiettivo di questa ricerca eseguita su un campione di 1100 studenti della Svizzera tedesca era quello di stabilire se l'affinità tra opzione specifica e materia di studio scelta all'università influenza il successo accademico. I risultati mostrano che chi studia materie affini all'opzione specifica non ottiene voti superiori di chi studia materie non affini all'opzione specifica. I risultati vengono discussi alla luce dell'obiettivo principale della formazione liceale, che è quello di garantire l'idoneità generale agli studi universitari.

Parole chiave: Successo accademico, transizione liceo-università, maturità, idoneità generale agli studi universitari, diploma di maturità, scelta della materia di studio, scelta dell'opzione specifica

\section{From specific subject matters at upper secondary school to university subject matters: Does studying a university subject matter that is related to a former specific subject matter result in higher academic success?}

\section{Summary}

Upper secondary schools in Switzerland deliver teaching in so called "specific subject matters", with the aim of providing an in-depth preparation for related university subject matters. Based on a sample of 1100 students from the German-speaking part of Switzerland, this study examines whether completing a related specific subject matter at the upper secondary school affects academic success. The results show that university students who study a subject matter that is related to their former specific subject matter do not perform better than those with a subject matter that is unrelated to their specific subject matter. The findings are discussed in view of the objective of the upper secondary school, which is to provide general abilities for further academic studies.

Keywords: Academic success, transition secondary school - university, general ability to study, general qualification for university entrance, choice of field of study, choice of special subject 
